\title{
Ventricular Arrhythmias in Acute Coronary Artery Ligation in Dogs: Electrophysiological Mechanism and Its Relation to the Severity of Myocardial Ischemia
}

\author{
Satoshi Ogawa, M.D., Yoshiro Nakamura, M.D. \\ LEONARd S. Dreifus, M.D*, and Elieser Kaplinsky, M.D*
}

\begin{abstract}
Analysis of conduction characteristics within the ischemic myocardium and the incidence of ventricular arrhythmias for the first 30 minutes of coronary artery ligation identified two phases of ventricular arrhythmias with different electrophysiólogical mechanisms. The first phase appearing during 2-10 minutes (immediate ventricular arrhythmias: IVA) was closely related to the degree of epicardial delay and thus considered to be initiated by reentry within the ischemic epicardial zone. In contrast, the second or delayed phase (12-30 minutes, delayed ventricular arrhythmias: DVA) of ventricular arrhythmias appearing independently from epicardial delay was suggested to originate from the subendocardial Purkinje network, since endocardial activation of the ischemic zone consistently preceded the QRS complexes of ventricular arrhythmias.

Measurements of myocardial adenosine triphosphate (ATP) contents demonstrated that in animals developing a profound myocardial ischemia by 3-5 minutes, epicardial delay was not observed and thereby IVA was not initiated. In contrast, epicardial delay and IVA were associated with a significant, but milder or slower ischemic process. At the time of DVA, myocardial ATP contents were at extremely low levels for both groups with and without DVA, supporting the concept that DVA is not originated from the ischemic myocardial cells, but from Purkinje fibers depressed secondarily by surrounding ischemic myocardial cells.
\end{abstract}

$\mathbf{P}$ ATHOPHYSIOLOGY of malignant ventricular arrhythmias in ischemic heart disease has been extensively studied in the past few years. To date it is believed that reentry movement of excitation within the ischemic myocardium plays a major part in generation of ventricular arrhythmias in the early post-ligation period $(0-30$ minutes) $!^{1-5}$ In contrast, less emphasis has been

Key Words:

Ventricular arrhythmia

Ischemic heart disease

Electrophysiology

Myocardial metabolism

placed on the role of specialized conducting fibers in this period since subendocardial Purkinje fibers are less susceptible to ischemia, probably as a result of the paucity of myofibrils 6 and/or an increased content of glycogen? However, it has been demonstrated in recent studies that the Purkinje network may participate in the reentry pathways, attributing to the spread of reentrant impulses from the ischemic subepicardium to the normal myocardium 8,9 In addition, Purkinje fibers may be affected by local toxic accumulation of potassium, catecholamines, lactate and other metabolites that are leaking

Cardiopulmonary Division, Department of Medicine, Keio University School of Medicine, Japan

*Department of Cardiology, The Lankenau Hospital, Philadelphia, Pennsylvania, USA

Address for reprints: Satoshi Ogawa, M.D., Cardiopulmonary Division, Department of Medicine, Keio University School of Medicine, 35 Shinanomachi, Shinjuku-ku, Tokyo 162, Japan 
from the ischemic myocardial cells,$^{10}$ and thereby may gain the potential ability to become the site of reentry even in the early phase of ischemia.

It thus appears that the severity and the time course of myocardial cell ischemia following coronary artery ligation should have a critical relevance to the electrophysiological disturbances occurring in the working myocardial cells as well as the Purkinje system in the genesis of ventricular arrhythmias. This study is designed to elucidate the exact time course of the electrophysiological abnormalities and to try to correlate these changes with the severity of myocardial ischemia in the canine model.

\section{MATERIALS AND METHODS}

Two groups of mongrel dogs were studied following intravenous administration of sodium pentobarbital $(30 \mathrm{mg} / \mathrm{kg})$ and under artificial ventilation. The heart was exposed through left thoracotomy and suspended in a pericardial cradle. The left anterior descending coronary artery was dissected and freed from its bed 1-2 $\mathrm{cm}$ from its origin. In 41 dogs of group $\mathrm{A}$, 20-30 close-bipolar, Teflon-coated plunge electrodes were impaled at various endocardial and epicardial points in the expected areas of the ischemic, border and normal zones, as previously described 8,9 The local electrograms were recorded with the frequency response of 50-1000 Hertz by means of a "composite" electrode for each zone or by a single electrode on a HewlettPackard eight-channel photographic recorder (model 4578).

After recording the control data the coronary artery was ligated in one stage. Sequential changes of local electrograms and the incidence

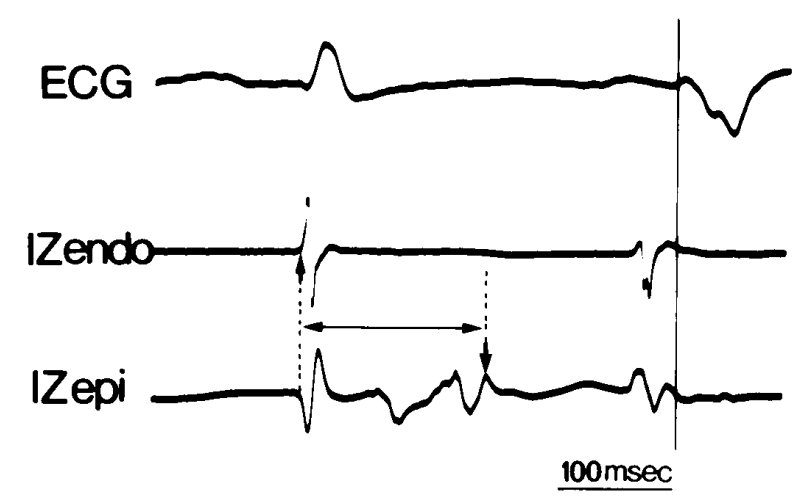

Fig.1. Electrocardiogram (ECG) and composite electrograms from ischemic zone endocardium (IZendo) and epicardium (IZepi) recorded 2 minutes post-ligation. of ventricular arrhythmias were analyzed for at least 30 minutes in all experiments. The degree of epicardial delay in the ischemic zone was quantitated by measuring the interval between the onset of the endocardial (or Purkinje) electrogram and the last distinct and sharp activity of the epicardial electrogram from the ischemic zone (Fig. 1). Dogs were considered arrhy thmiafree if they had less than two ventricular premature depolarizations per minute. Three successive ventricular depolarizations constituted an episode of ventricular tachycardia.

In 60 group B dogs, coronary blood flow was gradually reduced with a ligature constrictor. Blood flow reduction was $20-49 \%$ in 6 dogs, $50-74 \%$ in 8 dogs, $75-99 \%$ in 11 dogs and $100 \%$ in 35 dogs. Composite electrograms were recorded with 7-8 plunge electrodes from the ischemic epicardium and one from the endocardium. In 35 dogs with $100 \%$ occlusion, a transmural biopsy of the ischemic myocardium was performed in the area of recording at various times following ligation. A biopsy specimen was quickly compressed with a modified Aullenburgs' forcept which had been precooled in liquid nitrogen. This biopsy procedure took less than 15 seconds. The adenosine triphosphate (ATP) content was quantitated by Warburg's method 11 and was used as an index of myocardial ischemia. The biopsies were performed at 3-5 minutes postligation in 23 dogs and at $13-20$ minutes in 12 dogs.

\section{RESULTS}

\section{Time course of epicardial delay and evolution} of ventricular arrhythmias

Immediately following coronary artery ligation, remarkable changes in the local electrograms developed in the central ischemic zone epicardium (IZepi). Within 1-2 minutes the IZepi electrogram rapidly reduced its amplitude and fragmented into irregular, low amplitude spikes (Fig. 1). The degree of epicardial delay, the interval between the IZendo spike and the last distinct spike of the IZepi electrogram, progressively increased and reached its peak within 5 minutes (Fig. 2). The degree of epicardial delay gradually diminished in the ensuing 5-10 minutes and reached a plateau. This reduction in the degree of conduction delay was associated with a further decrease of the amplitude of the IZepi electrogram. The incidence of ventricular arrhythmias, in contrast, exhibited a distinct biphasic appearance in the first 30 


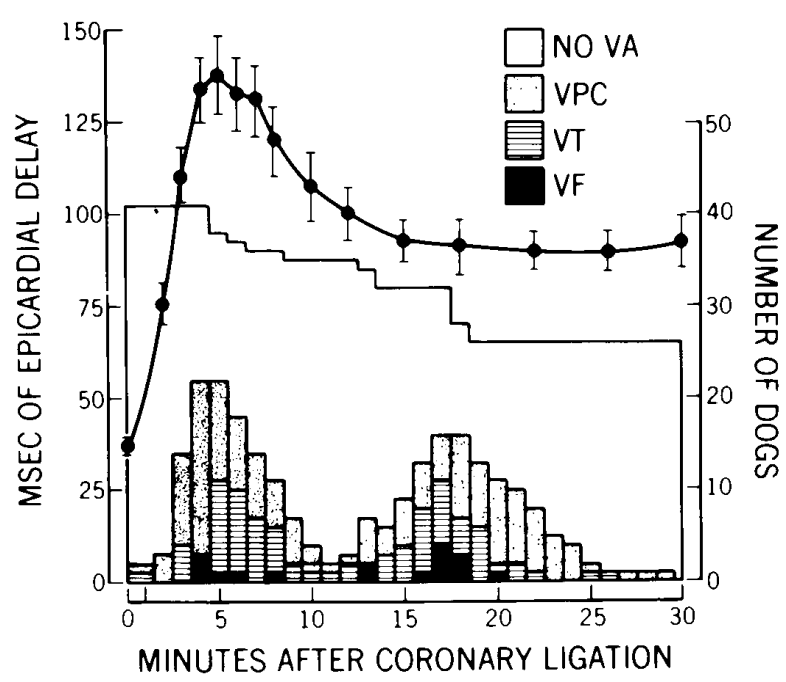

Fig.2. Epicardial delay and incidence of ventricular arrhythmias after coronary ligation in 41 dogs. (Reproduced from Circulation ${ }^{19}$ ) VPC=ventricular premature complexes, VA=ventricular arrhythmias, $\quad \mathrm{VT}=$ ventricular tachy cardia, VF=ventricular fibrillation. Same abbreviations are used in following figures. (by permission of the American Heart Association, Inc.)

minutes following ligation. The first phase of ventricular arrhythmias (immediate ventricular arrhythmias: IVA) began at 1-2 minutes. The frequency of the IVA rapidly increased in the following minutes and reached a peak incidence of $54 \%(22 / 41)$ at $4-5$ minutes and disappeared over the next 5-10 minutes. This time course of IVA was closely related to the rapid increase and subsequent decrease in the IZepi delay. The second surge, or delayed ventricular arrhy thmias (DVA), appearing independently from the epicardial delay, reached a peak incidence of $49 \%$ (16/33) during 17-18 minutes and declined gradually thereafter.

A representative case showing both IVA and DVA is presented in Fig. 3. It is clearly shown that appearance and disappearance of IVA was exclusively determined by the degree of the IZepi delay. The premature ventricular complexes during IVA were frequently preceded by a Wenckebach-type of conduction delay in the ischemic epicardium with fragmentation spreading into diastole (Fig. 4). Furthermore, during tachycardia the delay and fragmentation produced continuous activity in the epicardial electrogram bridging the diastolic intervals. In contrast, DVA abruptly began following a quiescent period without a marked epicardial delay (Fig. 3). In 16 dogs without IVA, epicardial delay rarely exceeded $100 \mathrm{msec}$ within 5 minutes post-

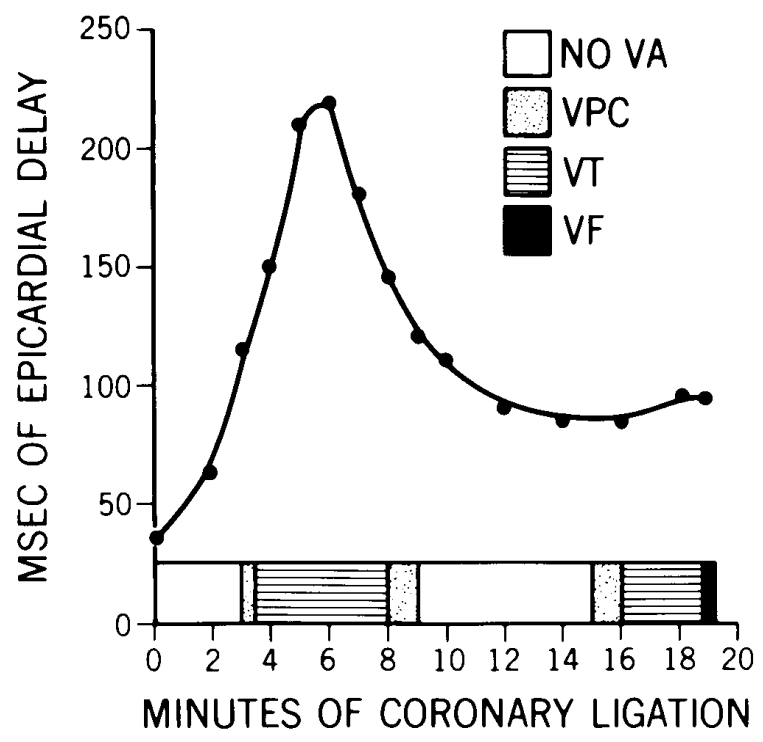

Fig.3. A representative sequence of epicardial delay in the experiment showing both immediate (IVA) and delayed (DVA) ventricular arrhy thmias.

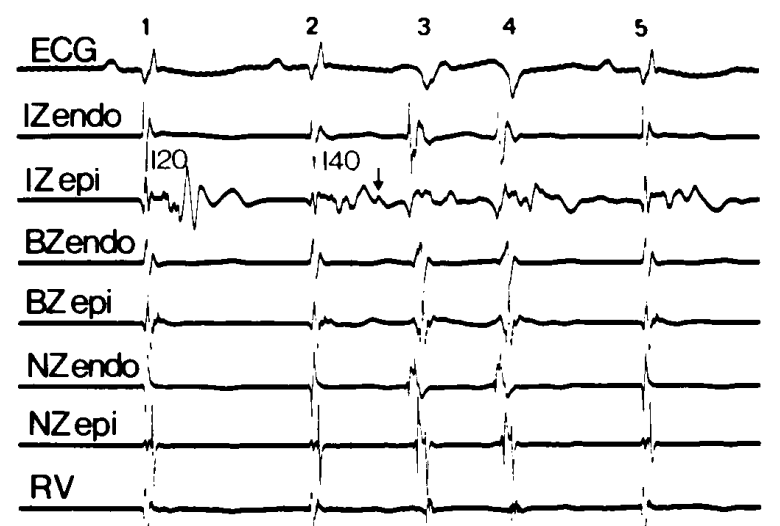

Fig.4. An example of immediate ventricular arrhythmia (IVA). The IZepi electrogram demonstrates Wenckebach-type conduction delay during normal sinus rhythm (120 msec in beat 1 and $140 \mathrm{msec}$ in beat 2) and premature ventricular complexes (beats 3 and 4) follow this marked delay producing diastolic bridging.

BZendo $=$ border zone endocardium

BZepi = border zone epicardium

NZendo $=$ normal zone endocardium $\mathrm{NZepi}=$ normal zone epicardium $\mathrm{RV}=$ right ventricle

Same abbreviations are used in the following figures.

ligation and DVA was a first manifestation of post-ligation arrhythmias (Fig. 5). As shown in Fig. 6, ventricular tachycardia during the phase of DVA was not associated with marked epicardial delay or diastolic bridging. It is interesting to note that endocardial activation or Purkinje 


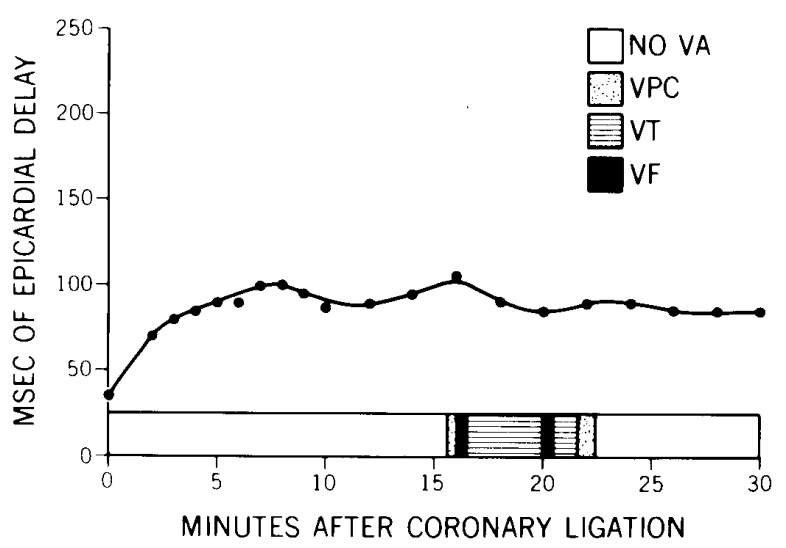

Fig.5. A representative sequence of epicardial delay in the experiment showing only DVA.

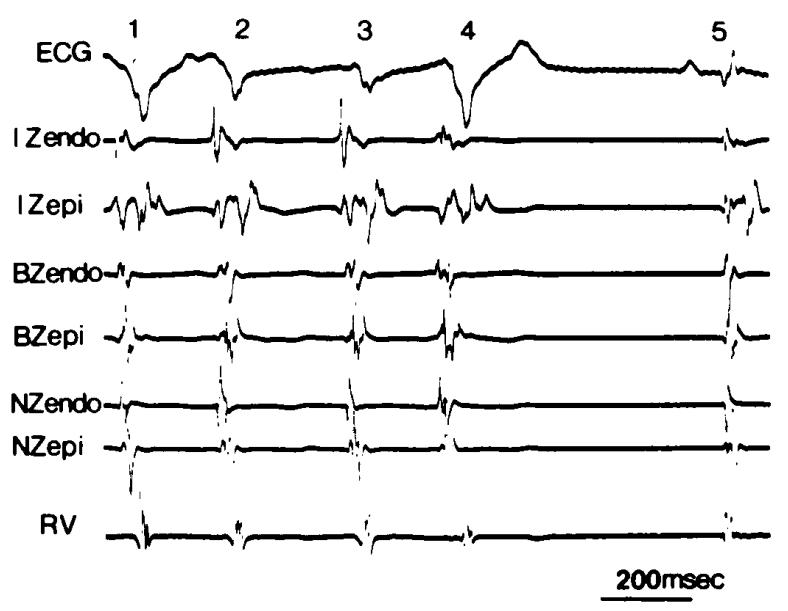

Fig.6. An example of ventricular tachycardia during the phase of DVA. Note the absence of marked epicardial delay or continuous activity on the IZepi electrogram. The IZendo electrogram is consistently inscribed at the onset of QRS complex and precedes all other electrograms.

activation in the central ischemic zone consistently preceded all other endocardial sites and was inscribed before the onset of QRS complexes in most instances of DVA.

\section{The severity of myocardial ischemia in rela- tion to the electrophysiological abnormalities}

Fig. 7 demonstrated maximal epicardial delay and the induction of IVA in the first 5 minutes of ligation in groups of dogs with variable degrees of coronary blood flow reduction. IVA associated with marked epicardial delay could be observed only in the cases of coronary flow reduction of more than $75 \%$. Although a few dogs of $75-$ $100 \%$ coronary flow reduction developed IVA in the absence of recordable epicardial delay, a

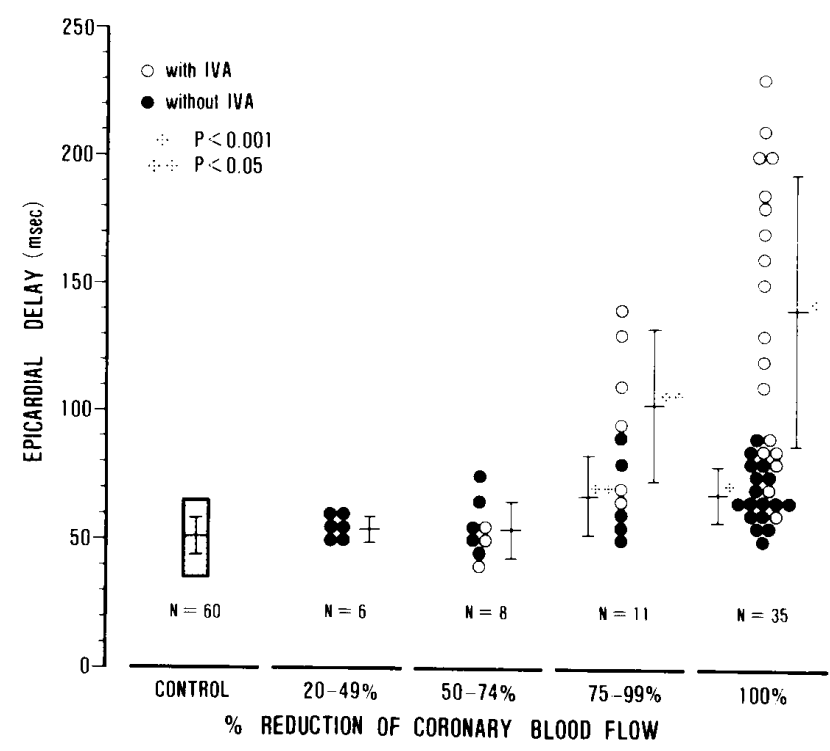

Fig.7. Maximal epicardial delays and incidence of ventricular arrhythmias within 5 minutes of coronary artery ligation of various degrees.

progressive diminution of the amplitude of the epicardial electrograms preceded the onset of IVA. Thus, it appears that the critical level of myocardial ischemia for electrophysiological abnormalities could be attained by coronary flow reduction of more than $75 \%$.

In order to determine the level of myocardial ischemia at the time of IVA and DVA, the myocardial ATP content was compared for dogs with complete coronary artery ligation. The maximal epicardial delay within 3-5 minutes following ligation was significantly more prolonged $(134.2 \pm 55.7 \mathrm{msec}, \mathrm{n}=13)$ in dogs with IVA than in dogs with no IVA $(68 \pm 13.8 \mathrm{msec}$, $\mathrm{n}=10)(\mathrm{p}<0.01) \quad$ (Fig. 8, left panel). Two examples were shown in Fig. 9. In both experiments, the amplitude of the epicardial electrogram became reduced 2-3 minutes following ligation with one (lower panel) demonstrating marked fragmentation and IVA, and the other (upper panel) no fragmentation and no IVA. The ATP content measured at 4 minutes was higher in the former than the latter. The measurement of the ATP content clearly indicated that the severity of myocardial ischemia in dogs with IVA was significantly less than those without IVA $(3.11 \pm 0.5 \mu \mathrm{mole} / \mathrm{g}$ vs $2.53 \pm 0.42$ $\mu$ mole $/ \mathrm{g}, \mathrm{p}<0.01$ ) (Fig. 8, right panel). Hence, animals suffering profound myocardial ischemia immediately following ligation (3-5 minutes) did not demonstrate epicardial delay and consequently did not develop IVA. 


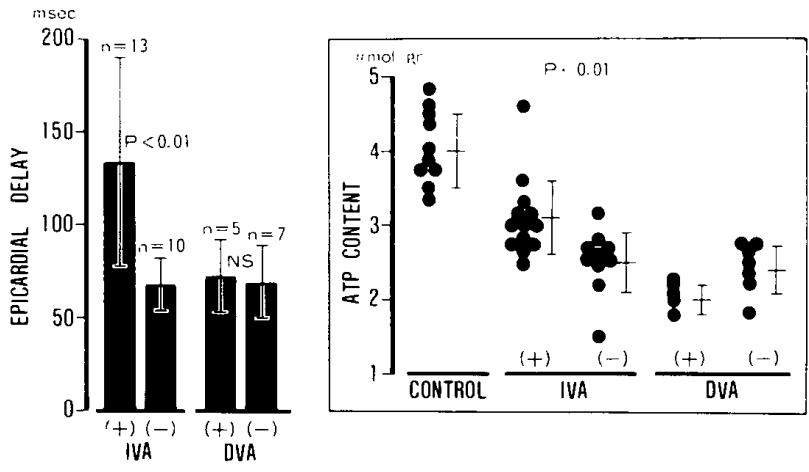

Fig.8. Comparison of epicardial delays and myocardial ATP contents at the time of IVA and DVA. See text for discussion.

In contrast, at the time of DVA, epicardial delay had decreased equally in both groups (Fig. 8 , left panel) and the ATP content in these dogs was markedly reduced irrespective of the presence or absence of DVA (right panel). These observations suggest that a majority of myocardial cells in the central ischemic zone were severely ischemic or dying and became electrophysiologically inexcitable at 13-20 minutes postligation. It appears that dogs with DVA had more profound myocardial ischemia than nonDVA dogs (ATP content: $2.06 \pm 0.16$ vs $2.43 \pm$ $0.32 \mu \mathrm{mole} / \mathrm{g}$ ). Of 5 dogs with DVA two also had experienced IVA within the first 10 minutes post-ligation and three no IVA.

\section{DISCUSSION}

The distinctive electrophysiological abnormalities of the specialized conducting fibers have been documented in the one day old canine infarction model by several groups of investigators! ${ }^{12-15}$ Friedman et al $!^{12,13}$ and Lazzara et al! ${ }^{14}$ found that surviving Purkinje fibers in the infarcted zone are partially depolarized and excessively automatic. These fibers generate action potentials with a reduced amplitude and an extraordinarily prolonged duration. Therefore, these cells have electrophysiological properties for both enhanced automaticity and reentry. Apart from these observations, the role of specialized fibers in acute phase of coronary ischemia (Harris phase 1) has yet to be fully elucidated. Lazzara et al! ${ }^{17}$ noted a diminution of the amplitude of the Purkinje electrograms recorded from the ischemic subendocardium within a few minutes after ligation. Furthermore, Purkinje cells, excised 20 minutes following ligation, were partially depolarized and generated action potentials with decreased amplitude, upstroke velocity and duration! ${ }^{17}$

However, these important observations have not been widely acknowledged because the Purkinje fibers are believed to be more resistant to the effects of anoxia than the working myocardium and may not be affected in the early
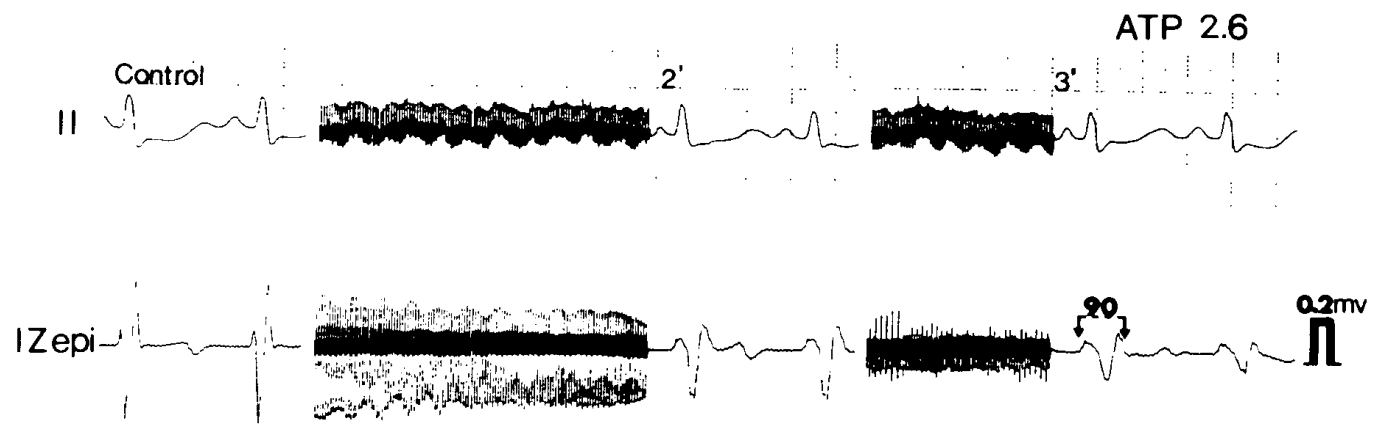

ATP 3.3

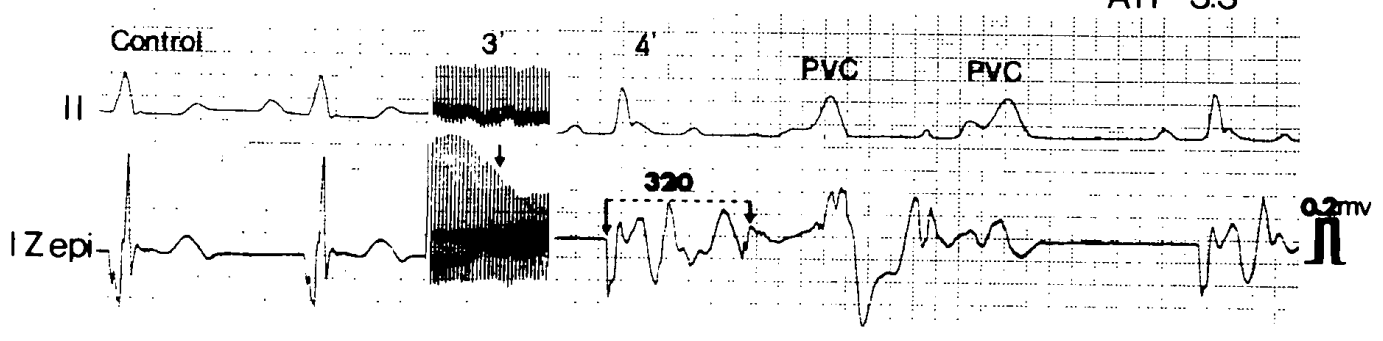

Fig.9. Two representative experiments showing a progressive diminution of the amplitude of IZepi electrograms with (lower panel) and without (upper panel) marked epicardial delay and IVA. PVC = premature ventricular complex. 
post-ligation period 18 Nevertheless, Kaplinsky et al ${ }^{8,9}$ recently emphasized the role of subendocardial Purkinje network in the central ischemic zone in the genesis of ventricular arrhythmias within the first few minutes after ligation (IVA). These investigators and others demonstrated that IVA was critically related to the degree of epicardial delay!-5,8,9 However, they believed that the Purkinje network could serve as the efferent reentry pathways, or could play a major role in transmitting the reentrant impulses from the ischemic subepicardium to the normal myocardium? Furthermore, induction of ventricular fibrillation was frequently preceded by the appearance of new fragmented endocardial electrograms during the phase of IVA. More importantly, as demonstrated in the present as well as previous studies ${ }^{19,20}$ Purkinje network in the ischemic zone appears to be a primary site for the initiation of a second surge of ventricular arrhythmias (DVA) in the period of 12-30 minutes following ligation. The DVA is consistently preceded by an early inscription of the ischemic subendocardial electrogram without manifest fragmentation or delay in any region of the myocardium (Fig. 6). Since enhanced automaticity is not yet apparent during this period, ${ }^{17,18}$ reentry within Purkinje network, probably microreentry as demonstrated by Wit et al.,21,22 appears to be an attractive mechanism for DVA.

Determination of myocardial ATP levels in this study could give some insight into the myocardial metabolism or ischemic process underlying the electrophysiological abnormalities seen during the acute post-ligation periods. Although the technique used in this study did not allow a sequential analysis of myocardial ATP in an individual dog, a comparison of the data obtained from a group of dogs could identify the basic process associated with myocardial ischemia in relation to the appearance of ventricular arrhythmias.

Basically, two patterns of electrophysiological events were identified in the first ten minutes following ligation (Fig. 9); one group of dogs demonstrated rapid increments of epicardial conduction delay leading to IVA and the other showing only diminution of amplitude without marked epicardial delay and IVA. It became evident that groups of dogs without IVA developed marked and significant myocardial ischemia 3-5 minutes after ligation. This process was associated with a rapid diminution of the ampli- tude of the local electrograms and was not necessarily associated with epicardial delay. In contrast, the severity of ischemia was significantly less in dogs with IVA as compared to the animals that did not demonstrate this early phase of ventricular arrhythmias. It was of interest that IVA and epicardial delay could only be demonstrated with marked reductions in coronary flow. Hence, it can be concluded that a significant, but milder or slower ischemic process could cause metabolic heterogeneity and produced marked conduction delay and IVA.

Since ATP levels were found to be extremely low when the specimen was sampled at 13-20 minutes post-ligation, irrespective of the presence or absence of IVA, myocardial ischemia was expected to progress continuously within the period of 5-13 minutes even in dogs with IVA. This process could attribute to the progressive decrease in epicardial delay and signify the disappearance of IVA, as demonstrated in Fig. 2. At the time of DVA, the myocardial ATP content was thus at extremely low levels for both groups with and without DVA. Electrophysiological findings of minimum epicardial delay and diminished amplitude of local electrograms during this period could possibly be explained by inexcitability of a majority of myocardial fibers on the basis of deep myocardial ischemia. These observations were in agreement with the histopathological study by Jennings et al., who demonstrated that myocardial cells initially became irreversibly injured approximately 20 minutes post-ligation. Thus, as far as the myocardial ATP content was used to judge the severity of ischemia, it appears likely that the central ischemic zone myocardium could no longer provide the metabolic or electrophysiological basis for the initiation of DVA. Remarkably, the ATP level was even more severely depressed in dogs with DVA than those animals that failed to demonstrate this late ventricular arrhythmias (Fig. 8). These findings would serve to further discredit the ischemic working myocardium as the site of reentry during DVA. Hence, the ischemic subendocardial Purkinje network could account for DVA.

\section{REFERENCES}

1. SCHERLAG BJ, HELFANT RH, HAFT JI, DAMATO AN: Electrophysiology underlying ventricular arrhy thmias due to coronary ligation. $\mathrm{Am}$ J Physiol 219: 1665, 1970

2. BOINEAU JP, COX JL: Slow ventricular activa- 
tion in acute myocardial infarction. A sourse of reentrant premature ventricular contractions. Circulation 48: 702, 1973

3. WALDO AL, KAISER G: A study of ventricular arrhythmias associated with acute myocardial infarction in the canine heart. Circulation 47: 1222, 1973

4. SCHERLAG BJ, EL-SHERIF N, HOPE R, LAZZARA R: Characterization and localization of ventricular arrhythmias resulting from myocardial ischemia and infarction. Circ Res 35: 372, 1974

5. EL-SHERIF N, SCHERLAG BJ, LAZZARA R: Electrode catheter recordings during malignant ventricular arrhythmias following experimental acute myocardial ischemia. Evidence for reentry due to conduction delay and block in ischemic myocardium. Circulation 51: 1003, 1975

6. JAMES TN, SCHERF L: Ultrastructure of myocardial cells. Am J Cardiol 22: 389, 1968

7. OPIE LH: Metabolism of the heart in health and disease. Part II. Am Heart J 77: 100, 1969

8. KAPLINSKY E, OGAWA S, KMETZO J, DREIFUS LS: Origin of the so-called right and left ventricular arrhythmias in acute myocardial ischemia. Am J Cardiol 42: 774, 1978

9. KAPLINSKY E, OGAWA S, BALKE W, DREIFUS LS: Role of endocardial activation in malignant ventricular arrhythmias associated with acute ischemia. J Electrocardiol 12: 299, 1979

10. OPIE LH, OWEN P, THOMAS M, SAMSON R: Coronary sinus lactate measurements in assessment of myocardial ischemia. Am J Cardiol 32: 295, 1973

11. LAMPRECHT W, TRAUTSCHOLD I: Adenosine5'-triphosphate: Determination with hexokinase and glucose-6-phosphate dehydrogenase. In Method of Enzymatic Analysis, ed by BERGMYER HV, New York Academic Press, 1963, p 2101

12. FRIEDMAN PL, STEWART JR, FENOGLIO JJ, WIT AL: Survival of subendocardial Purkinje fibers after extensive myocardial infarction in dogs: In vitro and in vivo correlation. Circ Res 33: 597, 1973

13. FRIEDMAN PL, STEWART JR, WIT AL: The spontaneous and induced cardiac arrhythmias in subendocardial Purkinje fibers surviving extensive myocardial infarction in dogs. Circ Res 33: 612, 1973

14. LAZZARA R, EL-SHERIF N, SCHERLAG BJ: Electrophysiological properties of canine Purkinje cells in one-day-old myocardial infarction. Circ Res 33: 722, 1973

15. SPEAR JF, MICHELSON EL, SPIELMAN SR, MOORE EN: The origin of ventricular arrhy thmias 24 hours following experimental anterior septal coronary artery occlusion. Circulation 55: 344, 1977

16. LAZZARA R, EL-SHERIF N, HOPE R, SCHERLAG BJ: Ventricular arrhythmias and electrophysiological consequences of myocardial ischemia and infarction. Circ Res 42: 740, 1978

17. LAZZARA R, EL-SHERIF N, SCHERLAG BJ: Early and late effects of coronary artery occlusion on canine Purkinje fibers. Circ Res 35: 391, 1974

18. BAGDONAS AA, STUCKEY JA, PIERA J, AMER ND, HOFFMAN BF: Effects of ischemia and hypoxia on the specialized conducting system of the canine heart. Am Heart J 61: 206, 1961

19. KAPLINSKY E, OGAWA S, BALKE CW, DREIFUS LS: Two periods of early ventricular arrhythmia in the canine acute myocardial infarction model. Circulation 60: 397, 1979

20. KAPLINSKY E, OGAWA S, KMETZO J, BALKE CW, DREIFUS LS: Intramyocardial activation in early ventricular arrhythmias following coronary artery ligation. J Electrocardiol 13: 1980

21. WIT AL, CRANEFIELD PF, HOFFMAN BF: Slow conduction and recentry in the ventricular conducting system. I. Return extrasystole in canine Purkinje fibers. Circ Res 30: 1, 1972

22. WIT AL, CRANEFIELD PF, HOFFMAN BF: Slow conduction and reentry in the ventricular conducting system. II. Single and sustained circus movement in networks of canine and bovine Purkinje fibers. Circ Res 30: 11, 1972

23. JENNINGS RB, SOMMERS HM, HERDSON PB, KALTENBACH JP: Ischemic injury of myocardium. Ann New York Acad Science 156: 61, 1969 